\title{
Profilaxia antenatal da isoimunização rh com imunoglobulina anti-D: efectividade e riscos
}

Rosália Rajão Braga*

\section{RESUMO}

Introdução: a administração pós-parto por rotina da Imunoglobulina (Ig) anti-D, bem como a sua administração aquando de eventos de risco para hemorragia transplacentária, diminuiu a incidência de isoimunização Rh de 17\% para 0,83-1,5\%. Para minimizar este risco prevê-se actualmente a sua administração antenatal por rotina.

Visto tratar-se de um hemoderivado é necessário ponderar os riscos inerentes à sua administração.

Objectivos: Rever a evidência disponível sobre a efectividade e riscos da administração por rotina da lg Anti-D durante a gravidez para profilaxia da Isoimunização Rh.

Métodos: Pesquisa de artigos de revisão, meta-análises, ensaios clínicos aleatorizados e controlados e normas de orientação clínica na Medline, sites BEM e índex de RMP, publicados em língua portuguesa, inglesa e espanhola, nos últimos 10 anos.

Conclusões: A profilaxia antenatal com Ig Anti-D (1.500 UI às 28 semanas ou $500 \mathrm{UI}$ às 28 e 34 semanas gestacionais) é efectiva na prevenção da Isoimunização Rh (SORT C). Nas doses utilizadas é insuficiente para causar anemia ou hemólise clínica no $\mathrm{RN}$ e o risco de transmissão de doenças infecciosas é baixo (SORT C). São necessários novos estudos com end-point centrado no paciente para avaliar o impacto desta medida na mortalidade e morbilidade do feto/recém-nascido.

Palavras-Chave: Imunoglobulina Anti-D; Isoimunização Rh; Gravidez; Eficácia; Riscos/Efeitos Adversos.

\section{INTRODUÇÃO}

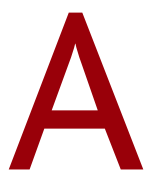

Doença Hemolítica do Recém-Nascido (RN) é uma condição na qual a semivida dos eritrócitos do feto/RN está diminuída por acção dos anticorpos maternos contra os antigénios por eles expressos.

A frequência de negatividade para o antigénio Rh é de aproximadamente $16 \%$ em Inglaterra e na Europa (segundo a Direcção-Geral da Saúde, em Portugal cerca de $15 \%$ das grávidas são Rh negativas). ${ }^{1}$ Esses indivíduos podem formar anticorpos anti-D quando entram em contacto com eritrócitos $\mathrm{RhD}$ positivos, quer através de transfusões sanguíneas (imunização em $90 \%$ dos casos) quer durante a gravidez ( $17 \%$ de casos de imunização).

O acontecimento sensibilizante na gravidez é, na maioria das vezes, a transfusão feto-materna de eritrócitos por hemorragia transplacentária, que vai aumentando de volume com a evolução da gestação.

Além do grupo Rh materno, paterno e fetal, existem vários factores que influenciam a probabilidade de isoimunização primária: volume da hemorragia transplacentária, coexistência de incompatibilidade $\mathrm{ABO}$ (que confere protecção parcial contra a isoimunização $\mathrm{Rh})$ e factores genéticos.

Em Inglaterra, a administração pós-parto por rotina da Imunoglobulina (Ig) anti-D, bem como a sua administração aquando de eventos de risco para hemorragia transplacentária durante a gravidez (aborto, gravidez ectópica, mola hidatiforme, hemorragia anteparto, técnicas invasivas de diagnóstico pré-natal, trauma abdominal, versão cefálica externa, descolamento prematuro de placenta normalmente inserida, dequitadura manual), diminuiu a incidência de isoimunização 
de $17 \%$ para $0,83-1,5 \%$. Os restantes casos de isoimunização ocorrem sobretudo por duas razões: não administração de Ig anti-D suficiente no momento correcto após eventos de risco (quer durante a gravidez, quer no pós-parto) e isoimunização durante a gravidez, resultante de hemorragia transplacentária «silenciosa». ${ }^{2}$

Visto tratar-se de um hemoderivado, é necessário ponderar os riscos inerentes à sua administração. Desde 1971, as unidades sanguíneas com antigénio de superfície do vírus da hepatite B (VHB) não são utilizadas para obtenção da Ig. O mesmo acontece desde 1985 e 1992 para o Vírus da Imunodeficiência Humana (VIH) 1 e 2, respectivamente. Desde o início da década de 90, a maior parte dos países desenvolvidos proibiu a utilização de unidades sanguíneas anti-Vírus Hepatite C (VHC) positivas. ${ }^{3}$

A prevenção antenatal por rotina da isoimunização Rh, tal como preconizado pela Direcção-Geral da Saúde na circular normativa no 2/DSMIA, de 15/01/2007, ${ }^{1}$ deverá ser implementada por excelência nos Cuidados de Saúde Primários. Desse modo, a autora considerou pertinente a realização do presente estudo, que teve como objectivo rever a evidência disponível sobre a efectividade e riscos da administração por rotina da Ig Anti-D durante a gravidez, para profilaxia da Isoimunização Rh.

\section{MÉTODOS}

Procedeu-se a uma revisão sistemática em bases de dados (Medline, TRIP) e sites de Medicina Baseada na Evidência (Cochrane, Bandolier, InfoPoems, Dare), de artigos de revisão sistemática, ensaios clínicos, meta-análises, normas de orientação clínica e pesquisa cruzada de artigos relacionados, publicados nos últimos dez anos em língua inglesa, portuguesa ou espanhola.

As palavras-chave utilizadas foram: Rho(D) Immune Globulin, Rh Isoimmunization, Pregnancy, Effectiveness, Risks/Adverse Effects.

Para avaliação da força de recomendação dos estudos foi utilizada a Strenght of Recommendation Taxonomy (SORT): A Patient-Centered Approach to Grading Evidence.

\section{RESULTADOS}

\section{Selecção dos artigos}

De 39 artigos encontrados, 26 cumpriam os critérios de inclusão, dos quais nove não integravam os objectivos desta revisão. Dos 17 artigos restantes, seis não se en-

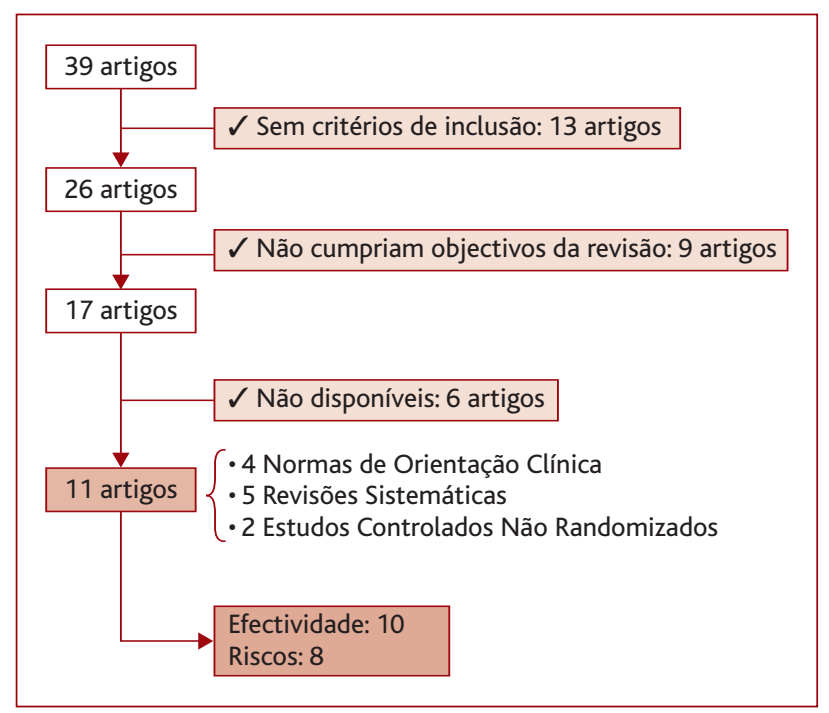

Figura 1. Metodologia.

contravam disponíveis.

Foram incluídos na presente revisão sistemática onze artigos: cinco Revisões Sistemáticas (RS), dois Es-

\begin{tabular}{|c|c|}
\hline Referência & Recomendações \\
\hline $\begin{array}{l}\text { U.S. Preventive } \\
\text { Services Task Force } \\
\text { (USPSTF), 20044 }\end{array}$ & $\begin{array}{l}\text { - A administração de } 1.500 \text { UI de } \\
\text { Imunoglobulina Rh(D) às } 24-28 \\
\text { semanas está recomendada para } \\
\text { todas as grávidas RhD negativas } \\
\text { não sensibilizadas. }\end{array}$ \\
\hline $\begin{array}{l}\text { National Health E } \\
\text { Medical Research } \\
\text { Council, } 2003^{5}\end{array}$ & $\begin{array}{l}\text { Profilaxia universal em mulheres } \\
\text { RhD-negativas, sem anticorpos } \\
\text { pré-formados, com Imunoglobulina } \\
\text { RhD ( } 625 \mathrm{UI}) \text { às } 28 \text { e } 34 \text { semanas } \\
\text { de gestação. }\end{array}$ \\
\hline $\begin{array}{l}\text { National Institute } \\
\text { for Clinical } \\
\text { Excellence, } 2002 \\
\text { (revisto em 2005) }^{6}\end{array}$ & $\begin{array}{l}\text { - A administração de } 2 \text { doses de } \\
500 \text { UI de Imunoglobulina Anti-D } \\
\text { às } 28 \text { e } 34 \text { semanas de gestação } \\
\text { parece ser tão eficaz como } 1 \text { dose } \\
\text { de } 1.500 \text { UI às } 28 \text { semanas. }\end{array}$ \\
\hline $\begin{array}{l}\text { BlueCross } \\
\text { BlueShield of North } \\
\text { Carolina, } 2002^{7}\end{array}$ & $\begin{array}{l}\text { Às } 28 \text { semanas repetir o Teste dos } \\
\text { Anticorpos nas grávidas } \\
\text { RhD-negativas não sensibilizadas. } \\
\text { - Se negativo, a grávida deve } \\
\text { receber Imunoglobulina anti-D } \\
\text { profilaticamente. }\end{array}$ \\
\hline
\end{tabular}


QUADRO II. Efectividade - Resultados (Revisões Sistemáticas)

\begin{tabular}{|c|c|c|}
\hline Referência & Resultados & Conclusões \\
\hline $\begin{array}{l}\text { Chilcott, } 2003^{8} \\
\text { Jones, } 2004^{9}\end{array}$ & 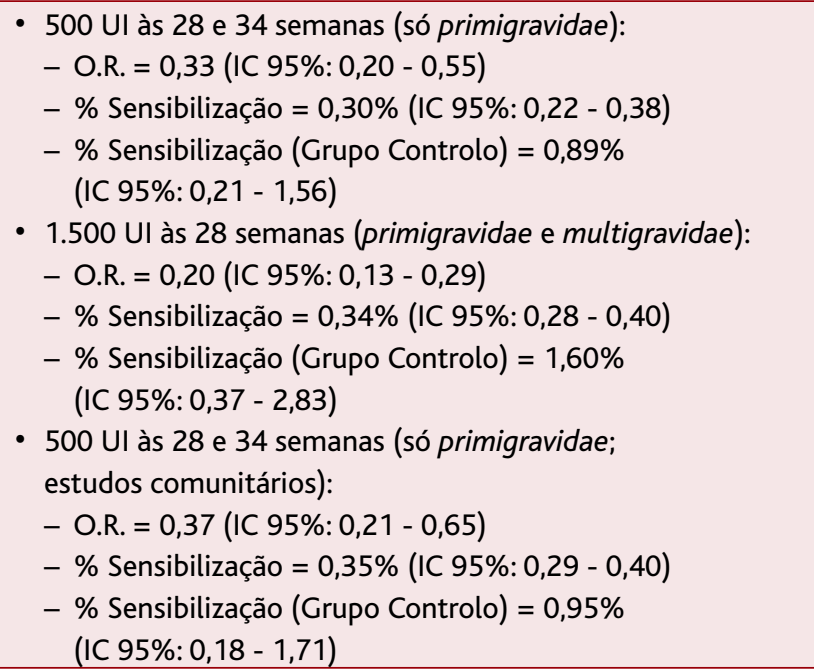 & $\begin{array}{l}\text { - } 2 \text { doses (às } 28 \text { e } 34 \text { semanas) } \\
\text { parecem ser mais efectivas do que } \\
\text { apenas } 1 \text { às } 34 \text { semanas. } \\
\text { - Não parece haver diferença } \\
\text { estatisticamente significativa entre } \\
2 \text { doses de } 500 \text { UI e } 1 \text { dose de } 1.500 \text { UI. } \\
\text { - } 2 \text { doses de } 250 \text { UI não são efectivas. }\end{array}$ \\
\hline Kee Fung, $2003^{10}$ & $\begin{array}{l}\text { - } 1.500 \text { UI Ig Anti-D devem ser administradas por rotina } \\
\text { a todas as grávidas Rh negativas não sensibilizadas às } \\
28 \text { semanas de gestação quando o tipo sanguíneo fetal } \\
\text { não é conhecido ou é Rh positivo. } \\
\text { Alternativa: } 2 \text { doses de } 500-600 \text { UI (28 e } 34 \text { semanas). } \\
\text { - Não é geralmente necessário repetir a dose de Ig anteparto } \\
\text { às } 40 \text { semanas de gestação (desde que a dose anterior não } \\
\text { tenha sido administrada antes das } 28 \text { semanas). }\end{array}$ & \\
\hline Neal, $2001^{11}$ & $\begin{array}{l}\text { - Duas modalidades efectivas: } 1 \text { dose de } 1.500 \text { UI } \\
\text { (às } 28 \text { semanas) ou } 2 \text { doses de } 500 \text { UI (às } 28 \text { e } 34 \text { semanas). }\end{array}$ & \\
\hline Urbaniak, $1998^{2}$ & $\begin{array}{l}\text { - } 1.500 \text { UI às } 28 \text { semanas ( } 3 \text { estudos): } \\
\text { - Redução de Aloimunização de } 1,6 \% \text { para } 0,18 \% \text { em } \\
\text { primigravidae e de } 2,2 \% \text { para } 0,42 \% \text { em multigravidae } \\
\text { ( } p<0,001) \\
\text { - Redução de } 1,8 \% \text { para } 0,27 \%(p<0,001) \\
\text { - Redução de } 1,86 \% \text { para } 0,32 \%(p=0,054) \\
\text { - } 1.500 \text { UI às } 28 \text { e } 34 \text { semanas (estudo em primigravidae): } \\
\text { - Redução de } 1,6 \% \text { para } 0,1 \%(p<0,001) \\
\text { - } 500 \text { UI às } 28 \text { e } 34 \text { semanas }(3 \text { estudos): } \\
\text { - Redução de } 0,9 \% \text { para } 0,16 \%(p=0,018) \\
\text { - Redução de } 0,95 \% \text { para } 0,32 \%(p=0,064) \\
\text { - Redução de } 1,19 \% \text { para } 0,17 \%(p=0,073) \\
\text { - } 1250 \text { UI às } 32 \text { semanas }(1 \text { estudo): } \\
\text { - Redução de } 1,6 \% \text { para } 0,38 \%(p=0,09) \\
\text { - } 250 \text { UI às } 28 \text { e } 34 \text { semanas }(1 \text { estudo): } \\
\text { - Redução de } 1,5 \% \text { para } 0,78 \%(p=0,395)\end{array}$ & $\begin{array}{l}\text { - } 2 \text { doses de } 250 \text { UI (às } 28 \text { e } 34 \text { semanas) } \\
\text { são insuficientes. } \\
\text { - Se se optar por uma só dose, } \\
\text { recomenda-se que seja às } 28 \text { semanas } \\
\text { ( } 34 \text { semanas é muito tarde). } \\
\text { - Pode-se optar entre } 2 \text { doses de } 500 \text { UI } \\
\text { (às } 28 \text { e } 34 \text { semanas) ou } 1 \text { única dose de } \\
1.500 \text { UI às } 28 \text { semanas }\end{array}$ \\
\hline
\end{tabular}




\section{QUADRO III. Efectividade - Resultados}

(Estudo Controlado Não Randomizado).

\begin{tabular}{l|l} 
Referência & Resultados \\
\hline Mackenzie, 199912 & - A profilaxia com 500 UI (às 28 e 34 \\
& semanas) diminuiu significativamente \\
& a isoimunização na gravidez \\
& subsequente quando comparada \\
& com controlos históricos (O.R. = 0,28; \\
& IC $0,14-0,53 ; p<0,0001)$ e \\
& contemporâneos (O.R. = 0,43; \\
& IC $0,22-0,86 ; p=0,02)$.
\end{tabular}

tudos Controlados Não Randomizados e quatro Normas de Orientação Clínica (NOC) - Figura 1.

\section{Efectividade da administração antenatal de rotina da Ig Anti-D}

Foi revista a evidência sobre a efectividade da administração antenatal de rotina da Ig Anti-D em dez artigos, incluindo quatro Normas de Orientação Clínica - Quadro I -, cinco Revisões Sistemáticas (sendo que uma corresponde à revisão da anterior) - Quadro II - e um Estudo Controlado Não Randomizado - Quadro III.

\section{Riscos da administração antenatal de rotina da Ig Anti-D}

Os oito artigos incluídos para revisão da evidência dis- ponível sobre os Riscos da Administração Antenatal de Rotina da Ig Anti-D englobaram duas Normas de Orientação Clínica - Quadro IV -, cinco Revisões Sistemáticas - Quadro V - e um Estudo Controlado Não Randomizado - Quadro VI.

\section{DISCUSSÃO}

Os estudos revistos são unânimes a considerar que a administração antenatal por rotina da Ig Anti-D, em grávidas Rh negativas, sem anticorpos IgG anti-D e sendo o pai RhD positivo ou desconhecido, é efectiva na prevenção da Isoimunização Rh. Contudo, os estudos até agora efectuados incluem Disease Oriented Eviden$c e$ (DOE), sendo necessário a realização de novos trabalhos com Patient Oriented Evidence That Matters (POEMS). Pode ser administrada uma dose de 1.500 UI $(300 \mu \mathrm{g})$ às 28 semanas ou duas doses de 500 UI (a primeira às 28 semanas e a segunda às 34 semanas de gestação). Em Portugal, só está disponível a dose de 1.500 UI (WinRhoSDF 1.500 UI, comercializada pela Baxter ${ }^{\circledR}$ ).

A administração da Ig Anti-D em todas as grávidas em risco versus só nas primigestas depende, em grande parte, da disponibilidade e custo da Ig (e não da sua efectividade).

A administração Antenatal por Rotina da Ig Anti-D não pode nem deve substituir a utilização da Ig nos casos definidos como potenciais eventos de hemorragia feto-materna.

Nenhum dos estudos revistos relatou anemia ou he-

\section{QUADRO IV. Riscos - Resultados (Normas de Orientação Clínica).}

\begin{tabular}{|c|c|c|}
\hline Referência & Efeitos no Feto/RN & Transmissão de Agentes Infecciosos \\
\hline $\begin{array}{l}\text { National Health \& } \\
\text { Medical Research Council, } \\
2003^{5}\end{array}$ & - Não há evidência de efeitos sobre o feto. & $\begin{array}{l}\text { - } \text { + s que receberam Profilaxia entre } 1970 \text { e 1994: } \\
\text { - 1,1\% com evidência de infecção HCV } \\
\text { - } 55 \% \text { com RNA-HCV+. } \\
\text { - Actualmente: selecção cuidadosa do dador, } \\
\text { testes ao plasma, inactivação viral por solvente/ } \\
\text { /detergente e nanofiltração } \rightarrow \text { sem evidência } \\
\text { de transmissão de doenças infecciosas. }\end{array}$ \\
\hline $\begin{array}{l}\text { National Institute for } \\
\text { Clinical Excellence, } \\
2002 \text { (revisto em 2005) }\end{array}$ & $\begin{array}{l}\text { - Não há evidência de que a exposição } \\
\text { in utero a Imunoglobulina Anti-D afecte } \\
\text { o sistema imunitário do feto/RN nem } \\
\text { que possa interferir na capacidade } \\
\text { reprodutiva dos RN do sexo feminino. }\end{array}$ & $\begin{array}{l}\text { O risco de infecção com um patogénio } \\
\text { conhecido (prião ou vírus) é extremamente } \\
\text { baixo }(<1 / 1 \text { milhão). }\end{array}$ \\
\hline
\end{tabular}




\section{QUADRO V. Riscos - Resultados (Revisões Sistemáticas)}

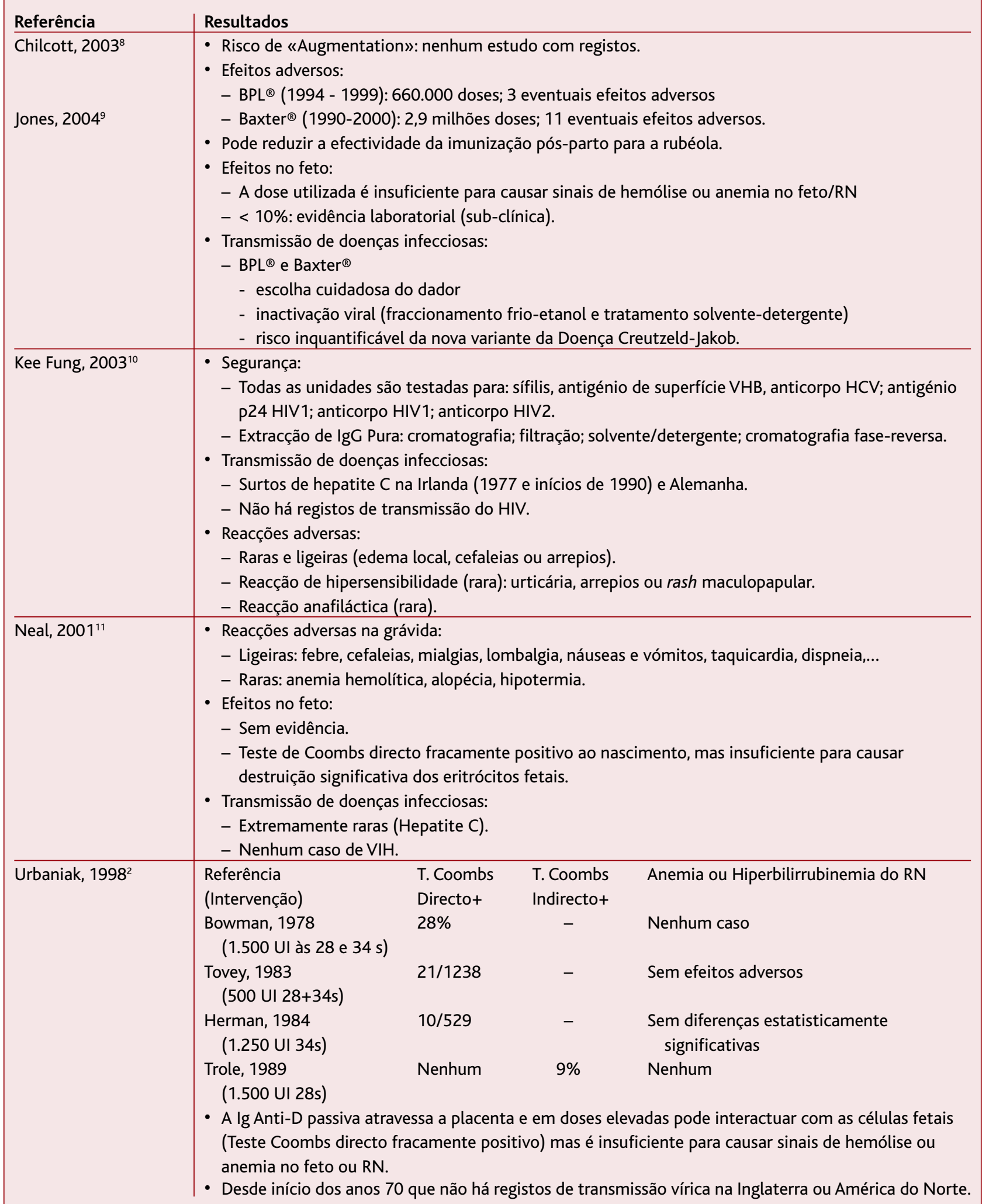




\section{QUADRO VI. - Riscos - Resultados (Estudo Controlado} Não Randomizado).

\begin{tabular}{|c|c|}
\hline Referência & Resultados \\
\hline $\begin{array}{l}\text { Maayan Metzger, } \\
2001^{13}\end{array}$ & $\begin{array}{l}\text { - Teste de Coombs directo positivo em: } \\
\text { - } 20 \% \text { dos filhos de mães que } \\
\text { receberam } 2 \text { doses } \\
\text { - 2,4\% dos filhos de mães que } \\
\text { receberam } 1 \text { dose. } \\
\text { - Efeitos na Feto/RN: } \\
\text { - Nenhum com sinais de hemólise } \\
\text { - Sem diferenças significativas } \\
\text { (hemoglobina, hematócrito e } \\
\text { percentagem de reticulócitos) } \\
\text { - Volume Globular Médio: } \\
\text { ligeiramente aumentado no } \\
\text { grupo que recebeu } 2 \text { doses. }\end{array}$ \\
\hline
\end{tabular}

mólise clínicas no RN e, após introdução dos actuais procedimentos na extracção de IgG Anti-D pura, não se detectaram casos de transmissão de doenças infecciosas conhecidas. Contudo, o risco potencial mantém-se na transmissão de vírus, identificados ou não, para os quais não há processos de rastreio disponíveis.

A necessidade de consentimento informado e esclarecido da grávida tem implicações nas atitudes e conhecimentos dos profissionais de saúde, com especial atenção do médico de família como principal cuidador.

Assim, tendo em consideração a demonstração em vários estudos da segurança da administração da Ig Anti-D, urge a realização de estudos com end-pointcentrado no paciente (por exemplo, avaliar o impacto da implementação desta medida na mortalidade do feto/RN por Isoimunização $R h$ ).

\section{CONCLUSÕES}

A profilaxia Antenatal com Ig Anti-D (1.500 UI às 28 semanas ou 500 UI às 28 e 34 semanas gestacionais) em grávidas RhD negativas, sem anticorpos IgG anti-D e sendo o pai RhD positivo ou desconhecido, é efectiva na prevenção da isoimunização Rh (SORT C).

A dose de Ig utilizada é insuficiente para causar anemia ou hemólise clínica no RN (SORT C).

Com os cuidados actuais na extracção da IgG Anti-D o risco de transmissão de doenças infecciosas é baixo

\section{(SORT C).}

É fundamental o consentimento informado e esclarecido da grávida para a administração antenatal por rotina da Ig Anti-D.

São necessários novos estudos com end-point centrado no paciente para avaliar o impacto desta medida na mortalidade e morbilidade do feto/RN.

\section{REFERÊNCIAS BIBLIOGRÁFICAS}

1. Divisão da Saúde Materna, Infantil e dos Adolescentes. Profilaxia da Isoimunização Rh. DGS. Circular Normativa N² 2/DSMIA, de 15/01/2007.

2. Urbaniak SJ. The scientific basis of antenatal prophylaxis. $\mathrm{Br}$ J Obstet Gynaecol 1998 Nov; 105 Suppl 18: 11-8.

3. Piazza M. Immunoglobulin transmits hepatitis C: true or false? Hepatology 1999 Jan; 29 (1): 299-300.

4. U.S. Preventive Services Task Force (USPSTF). Screening for Rh(D) incompatibility: recommendation statement. Rockville (MD): Agency for Healthcare Research and Quality (AHRQ); 2004.

5. National Health \& Medical Research Council. Guidelines on the prophylactic use of Rh D immunoglobulin (anti-D) in obstetrics. Canberra: National Blood Authority; 2003.

6. National Institute for Health and Clinical Excellence. Guidance on the use of routine antenatal anti-D prophylaxis for RhD-negative women. NICE Technology Appraisal Guidance N 41; 2002 May (review 2005).

7. BlueCross and BlueShield of North Carolina. Prenatal Practice Guidelines. AAP/ACOG Guidelines of Perinatal Care. 5th ed., 2002. Disponível em: www.bcbsnc.com/pdfs/clinicalguides/Prenatal.pdf [acedido em 13/01/2009].

8. Chilcott J, Lloyd Jones M, Wight J, Forman K, Wray J, Beverley C, et al. A review of the clinical effectiveness and cost-effectiveness of routine anti-D prophylaxis for pregnant women who are Rhesus-negative. Health Technol Assess 2003; 7 (4): iii-62.

9. Lloyd Jones M, Wray J, Wight J, Chilcott J, forman K, Tappenden P, et al. A review of the clinical effectiveness of routine antenatal anti-D prophylaxis for rhesus-negative women who are pregnant. BJOG 2004 Sep; 111 (9): 892-902.

10. Fung Kee Fung K, Eason E, Crane J, Armson A, De La Ronde S, Farine D, et al. Prevention of Rh alloimmunization. J Obstet Gynaecol Can 2003 Sep; 25 (9): 765-73.

11. Neal JL. RhD isoimmunization and current management modalities. J Obstet Gynecol Neonatal Nurs 2001 Nov-Dec; 30 (6): 589-606.

12. MacKenzie IZ, Bowell P, Gregory H, Pratt G, Guest C, Entwistle CC. Routine antenatal Rhesus D immunoglobulin prophylaxis: the results of a prospective 10 year study. Br J Obstet Gynaecol 1999 May; 106 (5): 492-7.

13. Maayan-Metzger A, Schwartz T, Sulkes J, Merlob P. Maternal anti-D prophylaxis during pregnancy does not cause neonatal haemolysis. Arch Dis Child Fetal Neonatal Ed 2001 Jan; 84 (1): F60-2.

Conflitos de Interesse: não assinalados

\section{ENDEREÇO PARA CORRESPONDÊNCIA}

Rosália Maria Rajão da Cunha Braga

Rua Cidade de Guarujá, 101 - 4490-503 Póvoa de Varzim

Tlm: 962682744

E-mail: rosalia.rajao@yahoo.com.br

Recebido em 07/07/2008

Aceite para publicação em 15/12/2008 


\section{ABSTRACT}

Introduction: routine after delivery administration of immunoglobulin (Ig) anti-D, as well as its administration in risk events for transplacental bleeding, decreased the incidence of $\mathrm{Rh}$ isoimmunization of $17 \%$ to $0,83-1,5 \%$. To minimize this risk it is now suggested its routine administration at antenatal period. Since this is derived from blood, it is necessary to consider the risks involved in its administration.

Objectives: to review the available evidence on the Effectiveness and Risks of the routine anti-D Ig administration during pregnancy for prophylaxis of Rh Isoimmunization.

Methods: search for articles from systematic review, meta-analyses, clinical trials, randomized controlled clinical studies and practice guidelines in Medline, MEB sites and index of RMP, published in Portuguese, English and Spanish, in the last 10 years. Conclusions: antenatal prophylaxis with Anti-D Ig ( $1.500 \mathrm{UI}$ at 28 weeks or $500 \mathrm{Ul}$ at 28 and 34 weeks of gestation) is effective in preventing Rh Isoimmunization (SORT C). The doses used are insufficient to cause anaemia or clinical hemolysis in newborns and the risk of transmission of infectious diseases is low (SORT C).

It would be advisable to do new studies with patient focused end-point to evaluate the impact of this measure on fetus/newborn mortality and morbidity.

Keywords: Rho(D) Immune Globulin; Rh Isoimmunization; Pregnancy; Effectiveness; Risks/Adverse Effects. 